\title{
Obesidade: técnicas cirúrgicas e indicações - revisão de literatura
}

\author{
Obesity: surgical techniques and indications - literature review \\ Natasha Mike Kawai ${ }^{1}$, Vitória Maria Leal Coelho ${ }^{1}$ Hamilton Cezar Rocha Garcia ${ }^{1}$
}

Resumo Objetivo: Realizar revisão sistemática de artigos que abordem sobre obesidade, técnicas cirúrgicas e indicações, publicados em língua inglesa e portuguesa. Método: Os artigos foram selecionados de acordo com o período de publicação de janeiro de 2013 a abril de 2018. Os descritores utilizados foram "obesidade", "cirurgia bariátrica", "técnicas cirúrgicas para obesidade", "grampeamento gástrico". As bases de dados utilizadas foram PUBMED, SCIELO, BVS e Biblioteca COCHRANE. Resultados: Foram encontrados 12 artigos que abordam as técnicas cirúrgicas mais utilizadas, atualmente, nos casos de obesidade e 4 artigos sobre as indicações para cada técnica cirúrgica empregada. Conclusão: Nos 16 artigos pesquisados, buscou-se a descrição e indicações das principais técnicas cirúrgicas utilizadas atualmente em pacientes obesos.

Descritores: cirurgia bariátrica; obesidade; obesidade abdominal; bandas gástricas.

Summary Objective: To carry out a systematic review of articles dealing with obesity surgical techniques and indications published in English and Portuguese. Method: The articles were selected according to the publication period from January 2013 to April 2018. The descriptors used were "obesity", "bariatric surgery", "surgical techniques for obesity", "gastric stapling", "gastric bands", "Bowel deviation". The databases used were PUBMED, SCIELO, BVS and Biblioteca COCHRANE. Results: We found 12 articles that deal with the most commonly used surgical techniques in cases of obesity and 4 articles on the indications for each surgical technique employed. Conclusion: In the 16 articles researched, we sought the description and indications of the main surgical techniques currently used in obese patients.

Keywords: bariatric surgery; obesity; abdominal obesity; gastric bands.

${ }^{1}$ Faculdade Metropolitana da Amazônia - FAMAZ, Belém, PA, Brasil

Fonte de financiamento: Nenhuma.

Conflitos de interesses: Os autores declaram não haver conflitos de interesse.

Recebido: Maio 29, 2018

Aceito: Junho 09, 2018

Trabalho realizado na Faculdade Metropolitana da Amazônia - FAMAZ, Belém, PA, Brasil. 


\section{Introdução}

A obesidade está associada ao aumento de mortalidade por várias causas, pois é um fator de risco agravante para doenças crônicas não transmissíveis como infarto do miocárdio, hipertensão arterial, diabete mellitus e também reflete o aumento de morte por câncer. A projeção Mundial da obesidade até 2025 é que cerca de mais de 700 milhões de pessoas estejam obesas. Em vista disso a OMS (Organização Mundial da Saúde) passou a considerar a obesidade como uma epidemia ${ }^{1}$.

Segundo a pesquisa do Ministério da Saúde realizada em 2017, apontou-se que em 10 anos a obesidade cresceu $60 \%$ no país. Os dados coletados indicam que mais de 50\% dos brasileiros estão acima do peso. Na região Norte o excesso de peso em adultos é $47.2 \%$; ou seja, quase metade da população não está no peso adequado ${ }^{1,2}$.

Existem diversos tratamentos disponíveis desde orientações alimentares, mudanças comportamentais até intervenção cirúrgica. As cirurgias bariátricas surgem como alternativa de tratamento quando os métodos anteriores não são efetivos ou de acordo com a indicação para cada paciente. São divididas em derivativas e não derivativas, cada uma apresentando características distintas ${ }^{2,3}$.

O excesso de peso acarreta alterações no estado de saúde, está relacionado ao aumento das doenças crônicas e pode levar a morte. No entanto, a prevenção é possível e eficaz, logo, é de suma importância que a redução da obesidade e consequentemente a diminuição de riscos associados, seja adotada como meta para saúde pública ${ }^{3}$.

As indicações para a cirurgia seguem critérios preestabelecidos, como o IMC (índice de massa corpórea) acima de $40 \mathrm{~kg} / \mathrm{m} 2$, ou com IMC maior que $35 \mathrm{KG} / \mathrm{M}^{2}$ e afetados por comorbidades que ameacem a vida e idade entre 18 e 65 anos. É importante ressaltar que o paciente não deve fazer uso de drogas ilícitas ou álcool ou apresentar quadros psicóticos ou demenciais ${ }^{3}$.

Os pacientes acima de 65 devem ser avaliados por uma equipe multiprofissional para uma avaliação criteriosa do risco-benefício (risco cirúrgico, presença de comorbidades, expectativa de vida e os benefícios do emagrecimento). Neste contexto, em alguns casos, a melhor indicação são os métodos cirúrgicos para proporcionar a correção da obesidade e melhoria na qualidade e expectativa de vida do paciente. ${ }^{3}$

\section{Método}

Foi realizada uma revisão sistemática da literatura, com o intuito de buscar artigos que apresentassem correlação com técnicas cirúrgicas e indicações para o tratamento da obesidade. Foram selecionados estudos publicados de janeiro de 2013 a abril de 2018. Os descritores utilizados foram "bariatric", "obesity", "surgical techniques for obesity", "gastric stomach stapling", "gastric banding", "bowel by pass" .As bases de dados utilizadas foram medical literature analysis and retrieval system (MEDLINE), Scientific Electronic Library Online (SCIELO), BVS (Biblioteca Virtual em Saúde) e COCHRANE. Foram estabelecidos os seguintes critérios de inclusão: artigos originais e de revisão de literatura publicados e incluídos na base nos últimos cinco anos contendo informações referentes a obesidade, técnicas cirúrgicas e indicações. Como critérios de exclusão, adotou-se não analisar artigos com informações incompletas acerca da obesidade, técnicas cirúrgicas e indicações.

\section{Resultados}

Seguindo a estratégia definida, a busca bibliográfica resultou em 22.871 artigos da base MEDLINE. Após aplicados os critérios de elegibilidade, de acordo com o objetivo da pesquisa e critérios de inclusão, 7 artigos foram selecionados. A busca na base de dados Cochrane resultou em 245 artigos com a utilização do descritor "surgical techniques for obesity" mas somente 2 foram selecionados. Na BVS 580 artigos foram encontrados porém apenas 1 foi selecionado (o descritor foi "técnicas cirúrgicas para obesidade"). Na base de dados Scielo 13 artigos foram encontrados com o descritor "técnicas cirúrgicas para obesidade" sendo que 5 foram selecionados. Todos os artigos estão dentro do período de janeiro de 2013 a maio de 2018. 


\section{Discussão}

As técnicas cirúrgicas bariátricas estão divididas em não derivativas (banda gástrica laparoscópica ajustável e gastrectomia vertical) e derivativas (derivação gástrica com reconstituição do trânsito intestinal em Y de Roux - ou bypass gástrico - e derivações biliopancreáticas à Scopinaro e duodenal switch) ${ }^{3}$.

As principais indicações formais para operações bariátricas são: idade de 18 a 65 anos, IMC maior ou igual $40 \mathrm{~kg} / \mathrm{m}^{2}$ ou $35 \mathrm{~kg} / \mathrm{m}^{2}$ com uma ou mais comorbidades graves relacionadas com a obesidade ${ }^{3}$.

As contraindicações normalmente abrangem causas endócrinas, dependência em álcool ou drogas ilícitas; doenças psiquiátricas graves sem controle além de risco anestésico e cirúrgico inaceitável (ASA-IV). Deve-se levar em consideração o risco-benefício e resultados com maior eficácia quando comparados a tratamentos clínicos anteriores já realizados pelo paciente (reeducação alimentar, exercícios físicos e comportamentais) $)^{3}$.

Diante da análise dos artigos desta revisão, pode se observar que a maioria das técnicas na atualidade são realizadas de maneira laparoscópica, para facilitar o pós operatório, evitar grandes perdas sanguíneas, menos complicações e diminuir a necessidade de Unidade de Tratamento Intensivo. ${ }^{4}$

\section{Técnicas cirúrgicas}

\section{Banda gástrica ajustável}

Trata-se de uma técnica realizada por via laparoscópica que pode ser reversível, porém apresenta um alto grau de insucesso, normalmente ocorrendo uma segunda abordagem cirúrgica. Esta técnica apresenta um melhor resultado quando acompanhada de mudança no estilo de vida e apresenta uma baixa mortalidade ${ }^{5,6}$.

\section{Balão intragástrico}

Este procedimento não é uma cirurgia mas é considerado um método invasivo realizado de maneira endoscópica, por isso foi avaliado nesta revisão de literatura. Este método consiste na colocação de um balão de maneira endoscópica permanecendo por até 6 meses. De acordo com a literatura ele consiste em um procedimento de risco baixo mas que apresenta um alto percentual de reganho de peso e que leva a uma segunda operação $0^{7,8}$.

\section{Gastrectomia vertical}

A gastrectomia Vertical resulta em adequada perda de peso e é considerada como uma opção de tratamento cirúrgico bariátrico definitivo. Quando comparados os estudos, a gastrectomia vertical apresenta um menor número de efeitos adversos. No estudo de Mendes GA et al, esta técnica se mostrou eficiente na melhoria da qualidade de vida dos pacientes, já no estudo de Colquitt JL et al., 3 a $4 \%$ precisaram ser reoperados afetando a qualidade de vida dos mesmos ${ }^{9}$.

\section{Derivação gástrica em Y de Roux}

Está e a técnica mais utilizada atualmente. Como efeito principal esta técnica causa uma saciedade precoce e a perda do excesso de peso é de aproximadamente $70 \%$. Deve-se garantir reposição de complexo vitamínico e mineral e de vitamina B12, bem como monitoramento dos níveis séricos de ferro, cálcio e vitamina $\mathrm{D}$. Também foi observado que a derivação gástrica exigiu um tempo de internação pós operatório maior entre as técnicas analisadas, e 7 a $24 \%$ dos pacientes pesquisados no estudo de Colquitt JL et al. tiveram que ser reoperados ${ }^{10-12}$.

\section{Derivação biliopancreática à Scopinaro}

Nesta técnica observa-se a perda de peso pós-operatória relacionada principalmente à má absorção lipídica e calórica. Por isso, essa técnica possui maior incidência de efeitos adversos como diarreia, flatus fétidos, desnutrição que pode ser grave e ameaçar a vida e deficiência de vitaminas lipossolúveis. A perda do excesso de peso oscila em torno de 80\%. A mortalidade é de cerca de 1\%. Esta técnica deve ser indicada com extrema cautela, pois se associa com complicações nutricionais extremas ${ }^{13,14}$. 


\section{Derivação duodenal switch}

Esta e uma derivação da técnica anterior com preservação de algumas áreas para minimizar os efeitos adversos da disabsorção acelerada. Mesmo assim efeitos adversos citados na derivação bilopancreática à Scopinaro acontecem porém de maneira mais branda. A perda de peso oscila em torno de $75 \%$ a $80 \%$, comparável à da Derivação gástrica em Y de Roux ${ }^{15,16}$.

\section{Considerações finais}

De acordo com o exposto, a perda de peso e a diminuição das comorbidades associadas à obesidade são evidentes nos pacientes submetidos à cirurgia bariátrica. Desse modo, as diversas técnicas apresentam indicações específicas para cada caso, dentre elas a gastrectomia vertical apresenta maior perda de peso em menos tempo, a banda gástrica ajustável tem menores riscos de complicações e a derivação gástrica em $Y$ de Roux é a técnica mais utilizada atualmente. Neste contexto, qualidade de vida e o controle da obesidade confirmam o caráter curativo e a eficácia do procedimento cirúrgico.

\section{Referências}

1. Associação Brasileira para o Estudo da Obesidade e da Síndrome Metabólica. Diretrizes brasileiras de obesidade 2016. 4. ed. São Paulo: ABESO; 2016.

2. Nassif PAN, Malafaia O, Ribas-Filho JM, Czeczko NG, Garcia RF, Ariede BL. Gastrectomia vertical e bypass gástrico em Y-Deroux induzem doença do refluxo gastroesofágico no pós operatório? Arq Bras Cir Dig. 2014;27(Suppl 1):63-8. http://dx.doi.org/10.1590/s01026720201400s100016. PMid:25409970.

3. Colquitt JL, Pickett K, Loveman E, Frampton GK. Cirurgia para obesidade. Cochrane Data System Rev. 2014;(8):CD003641. http://dx.doi. org/10.1002/14651858.CD003641.pub4.

4. Brasil. Ministério da Saúde. Cirurgia bariátrica por laparoscopia. Brasília: Comissão Nacional de Incorporação de Tecnologia no SUS CONITEC; 2017. 37 p. (Relatório de Recomendação, no. 249).

5. Devadas $\mathrm{M}, \mathrm{Ku}$ DJ. Conversional weight loss surgery: an australian experience of converting laparoscopic adjustable gastric bands to laparoscopic sleeve gastrectomy. Obes Surg. 2018;28(7):1902-9. http://dx.doi.org/10.1007/s11695-018-3128-9. PMid:29455406.

6. Rhaiem R, Maghrebi H, Rebai W, Makni A, Ksantini R, Ben Safta Z. Long term results of laparoscopic gastric band. Tunis Med. 2017;95(6):440-3. PMid:29512801.

7. Silvério AO, Rebelo BRR, Prudente CA, Martins COM, Oliveira PMC, Cardoso DMM, Resplande JCF, Siqueira Jr JT. Balão intragástrico como tratamento ponte para a cirurgia. GED Gastroenterol Endosc Dig. 2017;36(3):109-14.

8. Müller V, Fikatas P, Gül S, Noesser M, Fuehrer KT, Sauer I, et al. Nova técnica para cirurgia da obesidade: Plicatura intragástrica Single Port intragástrico (IGS-IGP) reduz em média 51\% do volume gástrico em modelo experimental. Arq Bras Cir Dig. 2017 jan-mar;30(1):60-4. PMid:28489173.

9. Mendes GA, Vargas GP. Qualidade de vida após gastrectomia vertical avaliada pelo questionário Baros. Arq Bras Cir Dig. 2017;30(1):14-7. http://dx.doi.org/10.1590/0102-6720201700010005.

10. Ells LJ, Mead E, Atkinson G, Corpeleijn E, Roberts K, Viner R, Baur L, Metzendorf M, Richter B. Surgery for the treatment of obesity in children and adolescents. Cochrane Data System Rev. 2015;(6):CD011740 http://dx.doi.org/10.1002/14651858.CD011740.

11. Navarrete S, Leyba JL, LI SN, Borjas G, Tapia JL, Alcázar R. Results of the comparative study of 200 cases: one anastomosis gastric bypass vs Roux-en-Y gastric bypass. Obes Surg. 2018 [ahead of print];1-6. http://dx.doi.org/10.1007/s11695-018-3224-x. PMid:29713942.

12. Orci $L$, Chilcott $M$, Huber O. Short versus long roux-limb length in Roux-en- $Y$ Gastric bypass surgery for the treatment of morbid and super obesity: a systematic review of the literature. Obes Surg. 2011;21(6):797-804. http://dx.doi.org/10.1007/s11695-011-0409-y. PMid:21479976.

13. Mahawar KK, Kumar P, Parmar C, Graham Y, Carr WL, Jennings N, et al. Small Bowel Limb Lengths and Roux-en-Y Gastric Bypass: a Systematic Review. Obes Surg. 2016;26(3):660-71. http://dx.doi.org/10.1007/s11695-016-2050-2. PMid:26749410.

14. Kalfarentzos F, Skroubis G, Kehagias I, Mead N, Vagenas K. A prospective comparison of vertical banded gastroplasty and Roux-en-Y gastric bypass in a non-superobese population. Obes Surg. 2006;16(2):151-8. http://dx.doi.org/10.1381/096089206775565096. PMid:16469216.

15. Srikanth MS, Oh KH, Fox SR. Revision to malabsorptive Roux-en-Y gastric bypass (MRNYGBP) provides long-term (10 years) durable weight loss in patients with failed anatomically intact gastric restrictive operations: long-term effectiveness of a malabsorptive Roux-en-Y gastric bypass in salvaging patients with poor weight loss or complications following gastroplasty and adjustable gastric bands. Obes Surg. 2011;21(7):825-31. http://dx.doi.org/10.1007/s11695-010-0280-2. PMid:20835895. 
16. Queiroz C, Sallet JA, DE Barros E Silva PG, Queiroz LD, Pimentel JA, Sallet PC. Application of BAROS' questionnaire in obese patients undergoing bariatric surgery with 2 years of evolution. Arq Gastroenterol. 2017;54(1):60-4. http://dx.doi.org/10.1590/s0004-2803.2017v54n1-12. PMid:28079242.

\section{Autor correspondente}

Natasha Mike Kawai

Faculdade Metropolitana da Amazônia - FAMAZ

Avenida Visconde de Souza Franco, $n^{\circ} 72$, Bairro do Reduto

CEP 66053-000, Belém, PA, Brasil

Tel.: (91) 99108-4191

E-mail: Medkawai21@gmail.com

\section{Informação sobre os autores}

NMK e VMLC são graduandas do curso de Medicina da Faculdade Metropolitana da Amazônia (FAMAZ).

HCRG é mestre em cirurgia e pesquisa experimental pela Universidade do Estado do Pará (UEPA); possui residência médica em cirurgia geral pelo Hospital Ophir Loyola; é docente dos cursos de medicina da Faculdade Metropolitana da Amazônia (FAMAZ) e da Universidade do Estado do Pará (UEPA).

\section{Contribuição dos autores}

NMK e VMLC foram responsáveis pela pesquisa e redação do artigo; HCRG foi responsável pela orientação, pesquisa e revisão do artigo.

Todos os autores leram e aprovaram a versão final submetida ao Pará Research Medical Journal. 